\author{
Jan Lazar \\ Université d'Ostrava \\ Université d'Opole
}

\title{
Vers une classification toponymique des pseudonymes en français tchaté
}

\section{Introduction}

Les échanges en situation de temps réel sur Internet mettent en contact des internautes simultanément connectés qui ont besoin de communiquer par l'intermédiaire de leur ordinateur. Ces conversations électroniques se déroulent dans différents salons qui sont considérés comme des groupes de discussion, plus ou moins durables, où les tchateurs entrent avec l'objectif de converser avec d'autres tchateurs de même âge (p. ex. tchats entre ados), de même ville (Paris, Lyonpoche), de même intérêt (ciné, sport), etc. Même si le thème des discussions est déjà « identifié » par le nom du salon, les tchateurs sentent le besoin de s'identifier eux-mêmes par des pseudonymes préalablement choisis. L'énorme richesse des pseudonymes qu'on peut observer dans ce type de discussion a attiré notre attention. L'objectif principal de cet article est de faire une analyse détaillée des toponymes formant des pseudonymes dans le contexte du français tchaté. Notre analyse repose sur un corpus que nous allons présenter dans le paragraphe suivant.

\section{Présentation du corpus}

Le choix d'un corpus équivalent représentait un pas d'une importance cruciale pour notre recherche. Étant donné que le réseau Internet dispose d'un grand nombre de tchats, nous avons défini plusieurs critères pour notre choix:

1. Le tchat doit être destiné à un public francophone et ses discussions doivent se dérouler en français. Ceci est une condition essentielle pour tout linguiste analysant la langue française telle qu'elle est employée par les locuteurs natifs. Les ats examinés sont tous placés sur un serveur français et ne sont pratiquement connus que dans le monde francophone. Nous en déduisons que la quasi-totalité 
des tchateurs est francophone et que le risque qu'un tchateur non francophone s'y glisse est largement éliminé.

2. Le tchat doit disposer d'un volume appréciable d'utilisateurs connectés (au total nous avons observé 1071 pseudonymes).

3. Le tchat doit être gratuit et public et il faut qu'il dispose de plusieurs salons de conversation. Étant donné que nous voulons observer notamment les discussions des jeunes internautes, le tchat doit disposer d'un salon de conversation pour les jeunes (Diskut - salle « moins de 18 ans », Adoskuat - salle « Tchat entre ados », Chat - salle «Ados »)

4. Il s'agit d'un tchat général et non modéré. Les discussions ne s'orientent pas sur un thème particulier et ne sont pas dirigées par un modérateur qui distribue les tours de parole

5. Les conversations des tchats sont librement disponibles à l'enregistrement.

D'après les critères mentionnés nous avons choisi trois tchats qui constituent notre corpus, il s'agit des tchats: Diskut, ${ }^{1}$ Adoskuat, ${ }^{2}$ Chat ${ }^{3}$.

Nous nous sommes connecté à chacun de ces trois tchats deux fois, à des dates et heures différentes, pour pouvoir capturer le matériel le plus équilibré possible. Pour entrer dans les salons destinés aux jeunes tchateurs, nous nous sommes créé l'identité fictive d'un jeune Parisien qui utilisait le nick Olomouc. Même si plusieurs tchateurs nous ont adressé la parole, nous n'avons engagé la conversation avec aucun d'entre eux et nous n'avons envoyé de messages ni public ni privé. Notre objectif était d'observer et d'enregistrer des conversations spontanées, sans que les tchateurs soient informés de notre recherche. Pendant nos six sessions, nous avons rassemblé au total 1071 pseudonymes à étudier.

\section{Pseudonyme ou nickname?}

Le mot pseudonyme est dérivé du mot grecque pseudōnumos, composé de pseudès qui signifie « trompé » et de onuma, qui signifie «nom». Il en résulte que le pseudonyme est un nom d'emprunt que celui qui le porte utilise pour exercer une activité sous un autre nom que son identité officielle. On peut constater que l'une des caractéristiques principales de ce type de communication est un anonymat absolu [Jandová, David 2006: 45]. Le pseudonyme représente donc un moyen grâce auquel le tchateur peut se créer une identité fictive et ainsi participer à la discussion dans n'importe quel salon de clavardage.

Le deuxième terme que l'on peut retrouver sur les tchats français est le mot anglais nickname qui signifie « surnom, sobriquet». Néanmoins, il faut préciser

\footnotetext{
${ }^{1} \mathrm{http}: / /$ www.discut.fr

${ }^{2}$ http://www.adoskuat.com/zone-chat.html

${ }^{3}$ http://www.chat-fr.org
} 
que dans le contexte du tchat, on ne peut pas considérer les mots nickname et surnom comme synonymiques. Il faut souligner que le surnom n'est pas choisi par la personne qui le porte, mais est attribué par un tiers, tandis que le nickname est choisi par la personne qui le porte. Étant donné que la communication sur Internet se trouve sous une forte influence de la langue anglaise, on peut constater que le mot nickname est souvent préféré par les tchateurs francophones au lieu du mot français pseudonyme [Pierozak 2003: 196].

Chaque personne qui veut participer à la communication synchrone sur les tchats doit impérativement choisir son pseudonyme dont la présence est générée automatiquement dans la marge dès que l'internaute s'exprime.

miissdu62 > cc tt lmonde recherche mec de 14-15 an du 62

charlotte $>$ la creve jai de la fievre

Ajoutons que le pseudonyme joue un rôle identitaire [Pierozak 2003: 196], car il représente toujours un tchateur concret. On note que les tchateurs restent remarquablement fidèles à leurs pseudonymes qui sont considérés comme un capital socio-discursif important dans cette communauté linguistique [Pierozak 2003: 218]. Il est impossible que deux tchateurs choisissent le même pseudonyme. Si le tchateur désire se servir d'un pseudonyme qui a été déjà choisi par un autre tchateur, il doit y faire au moins quelques transformations orthographiques (p. ex. Ostrava $\rightarrow$ O.s.t.ra.va., Ova...). Précisons que le tchateur peut aussi changer son pseudonyme en cours de connexion pour des raisons différentes. Ce changement est signalé dans la fenêtre principale par le logiciel de gestion du canal.

bogoss62620 s'appelle maintenant bogosscelib

Cherche-un-mec-entre-12-et-15 s'appelle maintenant seuleaenmourir.

L'énorme hétérogénéité que connaît cette marge discursive nous mène à en proposer une classification, notamment du point de vue toponymique.

\section{Pseudonymes toponymiques}

Les paragraphes qui suivent s'intéressent aux pseudonymes qui sont créés à la base d'un toponyme. Notre objectif principal est d'étudier comment les toponymes fonctionnent dans les pseudonymes et à quel point ils participent à la formation des pseudonymes en français tchaté.

Il est important de souligner que ce sont généralement les anthroponymes (notamment d'origine anglaise ou américaine) qui sont le plus souvent utilisés pour former des pseudonymes [Jandová, David 2006: 77]. Il est à noter que les pseudonymes avec un composant anthroponymique ont plutôt un statut de prestige [Jandová, David 2006: 77], tandis que le composant toponymique y ajoute 
une information pratique concernant l'origine du tchateur. Nous observons que, pour faciliter la communication entre les tchateurs, notamment dans les salons de rencontre, le composant toponymique se combine souvent avec les indications de l'âge ou du sexe (voire de l'orientation sexuelle). On peut en déduire que ce type de pseudonyme y fonctionne comme une petite annonce [Jadová, David 2006: 77].

Les pseudonymes contenant un composant toponymique ou une indication toponymique représentent $12,6 \%$ de notre corpus, c'est-à-dire 135 exemples. Ce pourcentage élevé est notamment élargi par la présence particulière des numéros de régions et départements français, qui s'ajoutent au pseudonyme anthroponymique (belange88, Cylia77, osmose72, nadine62).

Le département est une division administrative de la France qui a été créée le 22 décembre 1789. Les départements forment le second niveau de division territoriale de la République française et se regroupent en régions. Actuellement, la France se compose de 101 départements regroupés en 27 régions, y compris les régions et départements d'outre-mer. Il arrive souvent que le composant toponymique soit remplacé par le numéro, qui apporte une information précise sur l'origine du tchateur, alors un autre composant toponymique devient redondant. Si le tchateur ajoute à son pseudonyme le numéro 59 , comme le font par exemple les tchateurs Jujudu59 et Chipie59, il est évident que ces deux tchateurs proviennent du département 59 , c'est-à-dire le département du Nord. Ce type de pseudonyme représente $79 \%$ des cas de notre corpus (107 exemples), parmi tous les pseudonymes toponymiques observés.

Un autre type de pseudonyme se compose d'anthroponyme + préposition del from + toponyme qui peut être éventuellement complété par l'indication de l'âge ou une autre information. Néanmoins, dans notre corpus, ils représentent seulement $6 \%$ (8 exemples):

Juliendelyon-17, mariedeParis-cherche-un-mec, missfromlangdoc, from DIJON CyliedeMarseille, mimideMONTP, bébertdeParis, juliedelil

Un autre cas particulier parmi les pseudonymes toponymiques constitue les pseudonymes qui précisent l'origine ethnique des tchateurs francophones. Étant donné que la langue française n'est pas seulement la langue officielle de la République française, il arrive que les autres tchateurs francophones sentent le besoin de préciser leur origine. Nous avons trouvé 11 exemples (8\%) dans notre corpus.

Algérienne, algérien, marokn, marocen, guadaloupé, BELGE, misSSUISSE, unebelge, gadalupez, sexysuisse, AMEriken

Le dernier type représente les pseudonymes dont le composant toponymique est formé par un exonyme ou un endonyme. Il représente dans notre 7\% (9 exemples). 
NY (abréviation de New York), ALONDON, SAN Francisco, Hollywwod, Holywoud, LA (abréviation de Los Angeles), Bruxel!, Barcelona, Rome22

Puisqu'on ne peut pas connaître l'identité réelle des tchateurs, on peut seulement proposer une hypothèse qui explique le choix d'un pseudonyme contenant un exonyme ou un endonyme. La première motivation peut être le besoin d'indiquer le lieu où se trouve le tchateur au moment de sa connexion. Il se peut qu'un tchateur francophone séjourne dans un pays étranger et ressente le besoin de parler avec ses compatriotes et, en même temps, d'indiquer et de souligner son éloignement géographique (p.ex. ALONDON).

Le choix d'un endonyme peut être aussi motivé par le prestige dont bénéficient la culture et la civilisation anglo-américaine dans le contexte du discours électronique médié [Lazar 2012]. Le pseudonyme contenant un toponyme lié à la culture américaine (NY, SAN Francisco, Hollywood) est souvent senti comme plus prestigieux qu'un mot français et dans ce cas n'indique pas réellement l'origine du tchateur.

\section{Conclusion}

Les pseudonymes, mêlant souvent diverses caractéristiques, peuvent être décrits à partir de points de vue différents. L'intention de notre étude était de montrer comment les toponymes participent à la création des pseudonymes dans le contexte du français tchaté. Il ressort de ce qui vient d'être présenté que le moyen le plus courant qui introduit une indication toponymique est le numéro. Cette préférence reflète le système particulier de numérotation de régions et de départements français. Le tchateur choisissant le numéro recourt à cette pratique car elle correspond à la règle essentielle du discours électronique médié, c'est-àdire exprimer «un maximum avec un minimum» [Dejond 2002: 35].

Les autres indications toponymiques restent plutôt minoritaires et sont utilisées pour souligner les origines ethnique des tchateurs. Un cas particulier représente les endonymes, qui sont souvent liés à la culture anglo-américaine et, d'après notre hypothèse, bénéficient d'un statut d'exclusivité et n'indique pas la vraie origine du tchateur.

\section{Bibliographie}

Anis Jacques, 1999, Internet, communication et langue française, Paris.

Anis Jacques, 2002, L'écriture, théories et descriptions, Bruxelles.

Chovancová Katarína, 2008, Les discussion en direct sur internet (Énonciation et graphie), Banská Bystrica. 
Dejond Aurélia, Mercier Jacques, 2002, La cyberl@ngue française, Bruxelles.

Jandová Eva, 2006, Konverzace na WWW chatu, Ostrava.

Jandová Eva, David Jaroslav, 2006, Čeština na WWW chatu, Ostrava.

Lazar Jan, 2012, Les anglicismes dans le discours électronique médié, „Studia Romanica Posnaniesia”, 39/4, Poznań, 61-69.

Panckhurst Rachel, 2006, Le discours électronique médié: bilan et perspectives, [en:] Annie Piolat (éd.), Lire, écrire, communiquer et apprendre avec Internet, Marseille, 345-366.

Pierozak Isabelle, 2003, La variation à la marge sur internet: pseudo et (re)présentations, „Cahier du Français modern", 8, 195-222.

Jan Lazar

To a toponymic classification of nicknames in French chats

(Summary)

This article focuses on toponyms forming the nicknames used by Internet users, especially in the context of real time communication on the Internet, which means communication on chats. The main aim of this article is to suggest a classification of nicknames formed from a toponym, as well as to bring their exact quantification in this particular type of electronic communication. A huge richness of nicknames is illustrated by the concrete examples taken from the corpus.

Słowa kluczowe: Internet, czat, pseudonim, toponim, toponimia.

Keywords: Internet, chat, nickname, toponym, toponymy. 